\title{
CRYSTALLINE SYMMETRY AND SURFACE TENSION
}

\author{
Charles RADIN \\ Mathematics Department, The University of Texas, Austin, Texas 78712, USA
}

Received 4 September 1981

Received in final form 9 December 1981

\begin{abstract}
We analyze a model of interacting ions and show that surface tension breaks the crystalline symmetry of the ground state.
\end{abstract}

\section{Introduction}

The fundamental mechanisms causing crystalline symmetry in matter at low temperature and pressure are not yet understood ${ }^{1}$ ), though there has been some recent progress ${ }^{2-10}$ ).

In particular in ${ }^{4,6}$ ) we considered two-dimensional models of particles interacting through a two body force consisting of a hard core and short range attraction (modelling a molecular force). One expects the ground state of such a system to consist, away from boundaries, of a perfect crystal with each particle surrounded by six neighbors. The only difficulty is that for a finite system (at zero pressure) such a crystal would have a regular hexagonal boundary and a surface tension tending to make the boundary circular, while a circular boundary is incompatible with a perfectly crystalline interior. The problem then is whether or not the surface tension is strong enough to overcome the tendency to crystalline ordering. We showed it is not, but through delicate inequalities which do not give one a feeling for how the problem would turn out under other circumstances, such as ionic or metallic bonding, or in three dimensions. In fact it was conceivable that a simpler approach might have shown surface tension inherently incapable of affecting crystalline symmetry.

This paper consists of an analysis of another two-dimensional model, a type common in studying antiferromagnetism though we prefer to think of it as modelling ions. We show that its ground state has a natural crystalline symmetry which, however, is indeed broken by surface tension. Thus surface tension is now proven to be an important aspect of the crystal problem. 


\section{The stripe model}

Let $L$ be the triangular lattice in the plane, which is generated by two unit vectors, $v$ and $w$, which are separated by an angle of $\pi / 3$. The coordinates $a$ and $b$ of the point, $\mathbf{j}=a v+b w$, are denoted $(a, b)$. The model under consideration (which we call the stripe model) is an Ising-like model on $L$ with pair interaction between "charges" $S(j)$ and $S(k)$ at sites $j \neq k$ given by $J_{j k}[S(j) S(k)-1]$, where $J_{j k}$ equals 1 for nearest neighbors, equals $g>0$ for next nearest neighbors and otherwise equals 0 . The charges have values \pm 1 . A "state" is a function with domain a nonempty subset of $L$ and range in $\{ \pm 1\}$; not all positions in $L$ need be filled. We define a "bond of type 1 (resp. 2 )" in a state $C$ to be the closed line segment in the plane joining a pair of nearest (resp. next nearest) neighbors in the domain of $C$ which have opposite charges. For a state $C, G_{1}(C)$ (resp. $G_{2}(C)$ ) is the planar graph which is the union of all the bonds of type 1 (resp. 2) in $C . G_{2}(C)$ is naturally decomposed into three disjoint subsets $G_{2}^{j}(C)=G_{2}(C) \cap A_{j}, j=1,2,3$, where the $A_{j} \subseteq L$ are defined as follows: $A_{2}=v+A_{1}, \quad A_{3}=w+A_{1}$ and $A_{1}=$ $\{m(v+w)+n(2 v-w) \mid m, n$ in $Z\}$. Let $S$ be a doubly periodic state (i.e. invariant under translation by two independent vectors), so its domain is infinite though not necessarily all of $L$, and let $\left\{t_{n}\right\}$ be a sequence of similar, simple, closed, piecewise smooth curves in $R^{2}$ whose interiors $\left\{R_{n}\right\}$ are increasing and have union $R^{2}$. Let $G(S)$ be any one of the four graphs $G_{1}(S)$, $G_{2}^{j}(S)$. Define: $G^{n}(S)=G(S) \cap R_{n} ; N_{n}(S)=$ number of vertices in $G^{n}(S)$; $F_{n}(S)=$ number of faces in $G^{n}(S) ; a_{n}^{j}(S)=$ number of bonds in $G^{n}(S)$ bounding exactly $j$ faces, $j=0,1,2 ; B_{n}(S)=$ number of bonds in $G^{n}(S) ; F_{n, j}(S)=$ number of faces in $G^{n}(S)$ bounded by exactly $j$ bonds. (A bond "bounds" a face if the face is on precisely one side of the bond.)

Since $F_{n, 3}(S)=0$ for all $n$, summing over faces we have

$$
\sum_{i \geq 4} j F_{n, j}(S)=2 B_{n}(S)-a_{n}^{1}(S)-2 a_{n}^{o}(S) .
$$

From Euler's formula, $N_{n}(S)+F_{n}(S) \geqslant B_{n}(S)+1$, with equality if $G^{n}(S)$ is connected. We rewrite this as

$$
4 B_{n}(S) \leqslant 4 N_{n}(S)+\sum_{j=4} j F_{n, j}(S)+\sum_{j=4}(4-j) F_{n, j}(S)-4
$$

Combining (1) and (2), we have

$$
B_{n}(S) \leqslant 2 N_{n}(S)-\frac{a_{n}^{1}(S)}{2}-a_{n}^{0}(S)-2-\frac{1}{2} \sum_{j=4}(j-4) F_{n, j}(S) .
$$


Since $S$ is doubly periodic the following limits as $n \rightarrow \infty$ are well defined: $f_{j}=\lim F_{n, j}(S) / N_{n}(S)$;

$$
b(S)=\lim B_{n}(S) / N_{n}(S) ; \text { and } a^{i}(S)=\lim a_{n}^{j}(S) / N_{n}(S) \text {. }
$$

Then from (3) we have

$$
b(S) \leqslant 2-\frac{a^{1}(S)}{2}-a^{0}(S)-\frac{1}{2} \sum_{j \geqslant 4}(j-4) f_{j}(S) .
$$

We have proven the following lemma:

Lemma. If $b(S)=2$ then $G(S)$ is connected and $F_{n, j}(S)=0$ for $j \geqslant 5$ and all $n$.

Next we note that for the doubly periodic state $\tilde{S}$ :

$$
\tilde{S}(j)=\left\{\begin{array}{cll}
1, & j=(2 m, n), & m, n \text { in } Z, \\
-1, & j=(2 m+1, n), & m, n \text { in } Z .
\end{array}\right.
$$

$G_{1}(\tilde{S})$ and $G_{2}^{j}(\tilde{S})$ all have $b(\tilde{S})=2$. For a doubly periodic state $S$ we define the energy density as the limit, as $n \rightarrow \infty$, of the energy per particle of those charges in $R_{n} \cap$ domain $(S)$. (This is clearly independent of the specific family $\left\{R_{n}\right\}$.) From the noted property of $\tilde{S}$ above it follows that a doubly periodic state $S$ has minimum energy density if and only if $G_{1}(S)$ and all $G_{2}^{j}(S)$ have $b(S)=2$. Next we classify such $S$.

Theorem. A doubly periodic state $S$ has minimum energy density if and only if it is $\pm \tilde{S}$ or a rotation of either $\pm \tilde{S}$ by either $\pm \pi / 3$.

Proof. Using the lemma we see that of the vertices in any $G(S)$ no three nearest neighbors (with respect to the appropriate lattice: $L$ or $A_{j}$ ) can all have the same charge. We call this the "rule of three". Now consider $G_{2}^{1}(S)$ and assume the abutting rhombuses of which it is composed are not all translates of each other. (We will obtain a contradiction.) In particular assume, without loss of generality that $S(0,0)=S(1,1)=S(0,3)=1$, and $S(2,-1)=S(-1,2)=S(2,2)=-1$. Then for either value of $S(1,0)$ the rule of three applied to $G_{1}(S)$ determines consecutively the values of $S$ at the five other nearest neighbors of $(1,1)$ (clockwise if $S(1,0)=-1$, counter-clockwise if $S(1,0)=1)$. But it then follows that $S(1,0)=S(0,2)=S(2,1)$ contradicting the rule of three for $G_{2}^{1}(S)$. We have thus determined $G_{2}^{1}(S)$ up to rotations by $\pm \pi / 3$ and reversal of all charges. Finally note that once $G_{2}^{1}(S)$ is determined either choice of any other charge determines (using the rule of three) the rest of $S$, in conformity with the theorem and completing the proof.

If $S$ is any doubly periodic state and $\left\{R_{n}\right\}$ is as above, the charges of $S$ in $R_{n}$ 
have energy (as $n \rightarrow \infty)$

$$
E_{n}(S)=-p N_{n}(S)+q\left(N_{n}(S)\right)^{1 / 2}+o(1),
$$

where $q>0$ depends on the shape of the $R_{n}$ but $p>0$ does not. The Wulff shape ${ }^{11}$ ) for $S$ is the unique shape for the $R_{n}$ yielding the minimum value of $q$. For $S=\tilde{S}$ and $g=1 / 9$, a straightforward computation shows the Wulff shape is a rhombus with interior angles of $\pi / 3$ and $2 \pi / 3$ and stripes (i.e. lines of constant charge) parallel to the short diagonal; it yields the value $q=8 / 3$ (and $p=20 / 9$ ).

Now note that if two finite rhombuses of the crystal (i.e. restrictions of states satisfying the theorem) with differently oriented stripes are joined with common edge there is no loss of energy across the domain wall due to nearest neighbors but only due to next nearest neighbors. Define the state $S^{\prime}$ as follows. Set $S^{\prime}(a, b)=\tilde{S}(a, b)$ for $(a, b)$ in $L_{1} \equiv\{(a, b) \mid b \geqslant 0\}$. Then for the sets $L_{+}$and $L_{-}$which are rotations of $L_{1}$ by $\pm \pi / 3$ about $(0,0)$ we define $S^{\prime}$ on $L_{ \pm}$by rotation of $\mp \pi / 3$. Finally we define $\left\{R_{n}^{\prime}\right\}$ to be regular hexagons centered at $(0,0)$, so that, for small enough $g, S^{\prime}$ produces a smaller $q$ in (4) than does the perfect crystal $\tilde{S}$ with any shape $\left\{R_{n}\right\}$ (i.e. with the Wulff shape.) In particular, for $g=1 / 9, S^{\prime}$ with $\left\{R_{n}^{\prime}\right\}$ yields $q=3^{1 / 2}+(48)^{1 / 2} / 9 \simeq 2.50<8 / 3$ (and of course $p=20 / 9$ ).

\section{Conclusion}

Particularly because of the pertinence of symmetry groups, the use of infinite volume states in studying the origins of crystalline symmetry is a temptation which, however, must be handled cautiously to be relevant ${ }^{12}$ ). The surprising phenomenon seen above in the stripe model is that even though it has a unique (infinite volume) crystalline state $\tilde{\boldsymbol{S}}$ which minimizes the energy density in a convincingly optimal fashion and even though one is tempted to view the polycrystalline state $\boldsymbol{S}^{\prime}$ as a higher energy perturbation of $\tilde{S}$ because of its domain walls, when we consider finite sets of charges and allow them to form their own boundaries (zero pressure) it is $S^{\prime}$ that turns out to have lower energy; surface tension breaks the crystalline symmety of $\tilde{S}$. Clearly such finite size effects as surface tension must be accounted for in understanding the symmetry of models and matter, in particular when using an infinite volume formalism.

\section{Acknowledgements}

We gratefully acknowledge useful discussions with Clifford Gardner and partial support by NSF Grant MCS81-01596. 


\section{References}

1) G.E. Uhlenbeck, in Fundamental Problems in Statistical Mechanics, II, E.G.D. Cohen, ed. (John Wiley, New York, 1968).

2) C.S. Gardner and C. Radin, J. Stat. Phys. 20 (1979) 719.

3) G.C. Hamrick and C. Radin, J. Stat. Phys. 21 (1979) 601.

4) R.C. Heitmann and C. Radin, J. Stat. Phys. 22 (1980) 281.

5) B. Borden and C. Radin, J. Chem. Phys. 75 (1981) 2012.

6) C. Radin, J. Stat. Phys. 26 (1981) 365.

7) W.J. Ventevogel, Physica 92A (1978) 343.

8) W.J. Ventevogel and B.R.A. Nijboer, Physica 98A (1979) 274.

9) W.J. Ventevogel and B.R.A. Nijboer, Physica 99A (1979) 569.

10) H. Kunz, Ann. Phys. 85 (1974) 303.

11) C. Herring, Phys. Rev. 82 (1951) 87.

12) Ch. Gruber and Ph.A. Martin, Phys. Rev. Lett. 45 (1980) 853. 